\title{
Resaerch Paper: Cultural Issues in Understanding Obsessive-compulsive Disorder: Nature, Appraisals, and Control Strategies of Unwanted Intrusive Thoughts in Iranian Nonclinical Population
}

Giti Shams $^{1}\left(\mathbb{D}\right.$, Imaneh $_{\text {Abasi }^{*}}{ }^{\star} \mathbb{D}$, Youness Yousefi $^{1}$

1. Department of Psychiatry, Tehran University of Medical Sciences, Roozbeh Hospital, Tehran, Iran

2. Department of Clinical Psychology, School of Medicine, Shahid Beheshti University of Medical Sciences, Tehran, Iran.

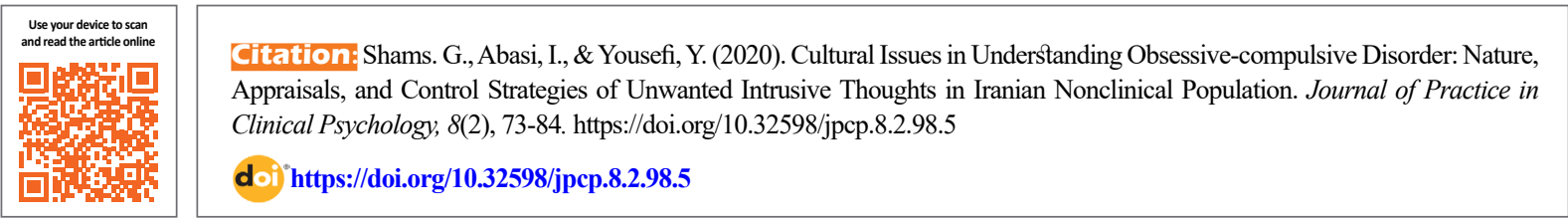

\section{(c) (1) (8)}

Article info:

Received: 10 Dec 2019

Accepted: 15 Feb 2020

Available Online: 01 Apr 2020
Keywords:

Obsessive-compulsive disorder, Unwanted intrusive thoughts, Control, Appraisal, Culture

\section{ABSTRACT}

Objective: Recent developments in cognitive models of obsessive-compulsive disorder posits that nearly everyone experiences unwanted intrusive thoughts, images, and impulses from time to time. The intrusions are not particularly a problematic issue; it is more probably an interpretation or attempts to control in maladaptive or unrealistic ways. So, the present article aimed to assess the nature, prevalence, appraisal, and control strategies of intrusions in the Iranian population.

Methods: We employed the international intrusive thoughts interview schedule in a sample of Iranian university students $(\mathrm{n}=50)$ selected by the convenient sampling method.

Results: Nearly all participants (100\%) reported experiencing at least one type of unwanted intrusive thoughts during the previous three months. The highest prevalence of Unwanted Intrusive Thoughts (UITs) belonged to the doubt category, and a large proportion of other UITs were also detected. However, the lowest prevalence of UITs was observed in the sexual and victim categories, respectively. In all of the UIT categories, support for the importance of removing and frequency had the highest mean levels. The highest mean level of endorsement for frequency was for the doubt category. Victim category had the highest mean level for the importance of removing and mean levels of interference/distress, and difficulty removing were higher in the contamination category. Participants endorsed intolerance of anxiety, uncertainty, and responsibility more as a reason that they noticed the thought. Participants were more likely to use thought replacement, distraction, self-reassurance, and avoidance as thought control strategies. The results are discussed in terms of Iranian culture.

Conclusion: Unwanted intrusive thoughts are reported by the majority of individuals in all countries, and significant cross-cultural differences, which are apparent in primary intrusive thought content, appraisals, and control strategies, are specifically related to obsessivecompulsive symptoms distress.

\section{* Corresponding Author:}

Imaneh Abasi, PhD

Address: Department of Clinical Psychology, School of Medicine, Shahid Beheshti University of Medical Sciences, Tehran, Iran.

Tel: +98 (21) 23031548

E-mail: abasi@sbmu.ac.ir; imanehabasi@gmail.com 


\section{Highlights}

- Nearly all participants reported experiencing at least one type of unwanted intrusive thoughts.

- The highest and lowest prevalence of unwanted intrusive thoughts belonged the doubt category and the sexual and victim categories, respectively.

- Participants reported more intolerance of anxiety, uncertainty, and responsibility as thought reappraisals.

- Participants used more thought replacement, distraction, self-reassurance, and avoidance as thought control strategies

\section{Plain Language Summary}

Unwanted intrusive thoughts are found in clinical obsessive and nonclinical people. They are essentially similar in content but are different in their processing. In other words, intrusions are not problematic, rather the ways we react to, interpret, appraise, and or attempt to control them can cause distress. So, the present study investigated prevalence, appraisal, and control strategies of intrusions in the Iranian population. The International Intrusive Thoughts Interview Schedule (IITIS) was implemented in a sample of Iranian university students $(n=50)$. Results showed nearly all participants reported experiencing at least one type of unwanted intrusive thoughts. The highest prevalence of Unwanted Intrusive Thoughts belonged to the doubt category, and the lowest prevalence of UITs was observed in the sexual and victim categories. Participants reported intolerance of anxiety, uncertainty, and responsibility more as a reason for noticing intrusions and they also were more likely to use thought replacement, distraction, self-reassurance, and avoidance for controlling intrusions. The results showed that unwanted intrusive thoughts, responses to these thoughts (thought reappraisals) and strategies to control them in Iranian- nonclinical population are almost similar to these in clinical OCD population. There were also some significant cross-cultural differences in prevalence, appraisal, and control strategies of unwanted intrusive thoughts.

\section{Introduction}

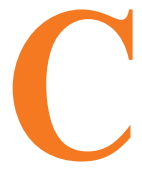

ulture may have a significant influence on the aspects of certain mental disorders (Nedeljkovic, Moulding, Foroughi, Kyrios, \& Doron, 2012). It has been suggested that obsessive-compulsive disorder (OCD) exhibits a certain degree of homogeneity across cultures (Del-Porto, 2001). However, OCD literature discusses influence of the culture on the clinical manifestations of this disorder. Recent developments in the field of OCD research indicate the commonality of intrusion thoughts between the normal population and patients with OCD. It also proposes that cognitive intrusions found in clinical obsessive and non-clinical people are essentially similar in content but are different in their processing (Abramowitz, 2006; Lipton, Brewin, Linke, \& Halperin, 2010; Moulding, Aardema, \& O'Connor, 2014).

Recently, OCD research has reported more details about the content and nature of unwanted intrusive thoughts (UITs) (Radomsky et al., 2014). OCD is a multi-faceted disorder that can manifest in a variety of symptom dimen- sions, including unacceptable thoughts, ruminations about morality, hoarding, etc. (Ruscio, Stein, Chiu, \& Kessler, 2010). Common obsessions include an excessive concern with dirt or germs, fears that harm may come to self or a loved one, and doubt about performing an action correctly, such as locking the door or shutting off the stove. Besides, the content of obsessions varies considerably across the disorder, with themes of aggression, blasphemy, and unacceptable sexual thoughts being the six most commonly identified forms of obsessions (Akhtar, Wig, Varma, Pershad, \& Verma, 1975; Rachman, 1998). The most common obsessional form is doubt, experienced by $75 \%$ of their sample. Dirt and contamination was the most prominent obsessional content, experienced by $46 \%$ of their sample. In another study, six forms of obsessions were identified that seemed to describe essentially the same behaviors as those of Akhtar et al. (1975), but with slightly different terminology (Khanna, Rajendra, \& Channabasavanna, 1988). Likewise, the factor structure of the Persian Padua Inventory (PPI) in the Persian sample exhibited eight content categories in OCD: Contamination obsessions, washing compulsions, checking compulsions, ordering compulsions, obsessional thoughts about harm to self/others, obsessional thoughts about violence, 
obsessional impulses to harm self/others, obsessional impulses to steal (Shams, Kaviani, Esmaili, Ebrahimkhani, \& Manesh, 2011). African-Americans reported more cleaning behaviors than European Americans in a non-clinical sample (Williams, Turkheimer, Schmidt, \& Oltmanns, 2005). They have suggested that contamination fear is the most common obsession in African-Americans with OCD. A similar pattern of studies conducted in the United States indicated similar results (Rasmussen \& Tsuang, 1986). Meanwhile, Radomsky et al. (2014) employed the international intrusive thoughts interview schedule (IITIS) to assess the nature and prevalence of intrusions in nonclinical populations. Their result demonstrated that nearly all participants $(93.6 \%)$ reported experiencing at least one intrusion thought during the previous three months. Doubting intrusions were the most commonly reported category of intrusive thoughts; whereas, repugnant intrusions (e.g. sexual, blasphemous, etc.) were the least commonly reported by participants (Radomsky et al., 2014). Cross-cultural research suggests that the cultural importance of an obsessional theme may help explain the high frequency of particular kinds of obsessions in a certain culture (Akhtar et al., 1975; Shooka, Al-Haddad, \& Raees, 1998).

Although most people admit that they have experienced UITs (Purdon \& Clark, 1993), the content of normally-occurring UITs is indistinguishable from obsessions (Rachman \& de Silva, 1978). These theories generally posit that the intrusions are not problematic, rather the ways we react to, interpret, appraise, and or attempt to control them can cause distress, fear, guilt, avoidance, compulsions (both overt and covert), as well as other symptoms, such as an increase in the frequency and or duration of the intrusions (Radomsky et al., 2014). Clinical experience and research have repeatedly indicated that underlying beliefs and appraisals are often intervening factors between obsessions and compulsions and they often play a role in maintaining OCD (Salkovskis, Forrester, \& Richards, 1997).

Regarding the appraisal and reaction strategies to these obsessions, intolerance of uncertainty in OCD may be most related to compulsions and ritualistic behaviors (Tolin, Abramowitz, Brigidi, \& Foa, 2003). Other researchers have found that intolerance of uncertainty was strongly correlated with both pathological worry and OCD symptoms (Dugas, Gosselin, \& Ladouceur, 2001). In an Iranian study, the domain that seems to be specific to OCD was a set of beliefs that revolves around the contention that is possible and necessary to control one's thoughts (Shams \& Milosevic, 2015). It was suggested that in Italian people, at least three domains (tolerance of uncertainty, control of thoughts, and perfectionism) were specific to OCD, whereas the importance of thoughts and responsibility barely discriminated clinically anxious individuals from non-clinical ones (Sica et al., 2004). Furthermore, some researchers reported similar findings in the study of British student participants. They found that the perfectionism and certainty (PC), as well as importance and control of thoughts (ICT), were independent predictors of specific OCD symptoms. However, they concluded that overestimation of threat, which predicted all types of OC symptoms, is likely relevant to multiple anxiety disorders and general anxiety traits (Myers, Fisher, \& Wells, 2008).

The perceived loss of control over thoughts is another key complaint of many individuals seeking treatment for OCD (Grisham \& Williams, 2009). Numerous research studies show that some thought control strategies are helpful, while others may contribute to preserving psychological distress (Abramowitz, Franklin, Schwartz, \& Furr, 2003; Belloch, Morillo, \& Garcia-Soriano, 2009). Among control strategies in OCD literature, distraction is considered one of the main controls. Distraction is more effective in reducing the frequency of the UITs than suppression (Salkovskis, Forrester, \& Richards, 1997). In contrast, individuals with OCD reported that they most frequently sought Excessive Reassuranceseeking (ERS) regarding potential general hurts, social threats, and perceived performance/competence. ERS is a common feature of OCD, other anxiety disorders, depression, and hypochondriasis. Evidence suggests that ERS is among the most common strategies used by OCD patients to diminish their obsessional thoughts and images (Freeston \& Ladouceur, 1997). Meanwhile, suppression or thought-stopping is another strategy of control and serves as a neutralization tool. It prevents the disconfirmation of the perceived negative consequences of the obsession (Rowa \& Purdon, 2003).

In the last three decades, research on intrusions has taken two broad pathways: The most common approach is questionnaire-based, whereas the other is more experimental, examining the impact of intentional mental control efforts on unwanted intrusive thoughts. Interestingly few studies have employed the interview format, some neglect that we think is serious given the complexity of intrusive thoughts. Several important conceptual and methodological weaknesses may arise with a questionnaire and experimental measurement of unwanted intrusive thoughts. Questionnaire measures of intrusions present individuals with a predetermined list of thought statements, and respondents are instructed to rate them for the frequency of occurrences. However, concerns have been raised about the truthfulness of these item responses (Brown \& Clark, 2014). 
When individuals indicate that they frequently experience an unwanted intrusive thought by endorsing an item statement, the questions are whether this reflects their actual memory of specific thought occurrences or an emotional state or self-identity that is congruent with the item content (Glass \& Arnkoff, 1997). Besides, the content validity of many intrusive thought questionnaires is problematic because they may contain a broad range of negative thought content that often includes general anxiety and depressive thought content (Clark \& Purdon, 1995).

Considering the literature, little is known about the impact of eastern as well as Iranian culture on OCD symptoms; however, research suggests some critical cultural variations. More investigations are needed to examine domestic or cross-cultural evaluations and determine the content, appraisal, and control strategy of obsessive thoughts in the normal population. Thus, the present study aimed to investigate six domains in IITIS as the nature and prevalence of intrusive thoughts across the culture of the Iranian non-clinical population. Also, we intended to assess interpretations/appraisals, and control strategies used by non-clinical populations, across Iranian culture.

\section{Methods}

\section{Study instruments}

The IITIS version 6 is a 101-item structured interview developed by the Research Consortium on Intrusive Fear (RCIF) to collect quantitative and qualitative information regarding individuals' experiences of appraisals and control strategies, and unwanted intrusive thoughts across seven content areas (i.e. contamination, harm/injury/ aggression, doubt, religious/immoral, sexual, victimization, and other intrusions). The detailed description of the history, development, and content of this instrument has been reported in another study (Radomsky et al., 2014). It is translated and back-translated by Shams into Farsi (Radomsky et al., 2014). The internal consistency of IITIS was 0.72 for contamination, 0.72 for harm/injury/aggression, 0.73 for doubt, 0.67 for religious/immoral, 0.78 for sexual, 0.70 for victimization, and 0.70 for other intrusive thoughts (Radomsky et al., 2014). Also, the internal consistency for appraisals, control strategies, and failure to control intrusive thoughts were 0.75 , 0.74 , and 0.72, respectively (Radomsky et al., 2014). The Cronbach $\alpha$ values based on Iranian data were calculated as 0.86 for contamination, 0.73 for harm/injury/ aggression, 0.88 for doubt, 0.77 for religious/immoral, 0.89 for sexual, 0.67 for victimization, and 0.83 for other intrusive thoughts. Besides, the Cronbach $\alpha$ values for appraisals, control strategies, and failure to control intrusive thoughts were $0.89,0.64$, and 0.46 , respectively.

\section{Study participants}

The study sample consisted of 50 (62\% females and $38 \%$ males) undergraduate students from Tehran University of Medical Sciences. They were recruited through a convenient sampling method. They were compensated with course credit or entry into a cash draw. Ethical approval was obtained from the Ethics Committee of the university (Ethical Code: IR.TUMS.MEDICINE. REC.1396.3199). The Mean $8 \pm \mathrm{SD}$ age of the total sample was $26.58 \pm 6.93,20.478 \pm 3.95$ years. About $38 \%$ of the participants were female, and $72 \%$ were single.

\section{Study procedure}

In the present study, the data collection and procedure are a part of studies from Radomsky et al. (2014). For detailed descriptions of the procedure, see Radomsky et al. (2014).

\section{Statistical analysis}

Continuous variables were presented as $\mathrm{Mean} \pm \mathrm{SD}$, and qualitative variables were reported through frequencies (percentages). The Chi-square test or Fisher exact test was used to compare the prevalence of UIT items between males and females. All statistical analyses were performed in SPSS, version 18 (SPSS Institute, Chicago, IL, USA). P-values less than 0.05 were considered statistically significant.

\section{Results}

The demographic characteristics of the study participants are tabulated in Table 1 . Overall, $100 \%$ of the participants reported at least one type of unwanted intrusive thought. The prevalence rates of the UIT categories are listed in Table 2. The highest prevalence of UITs belonged to the doubt category (80\%), and "other UITs" took a large proportion of intrusive thoughts (38.0\%). However, the lowest prevalence of UITs was observed in the sexual $(10.0 \%)$ and then victim $(16.0 \%)$ categories.

Table 3 presents the mean levels of UIT reports for associated frequency, interference/distress, the importance of removing, and difficulty removing. Generally, in all UIT categories, reports for the importance of removing and frequency have the highest mean values. The highest mean level of endorsement for frequency was for doubt category (Mean=3.13, SD=1.24). The victim category 
Table 1. Characteristic of the study participants (n: 50)

\begin{tabular}{|c|c|c|c|}
\hline Variables & Statuse & Categoris & Dates \\
\hline \multirow{2}{*}{ Age, $y$} & & Mean $\pm S D$ & $26.52 \pm 6.93$ \\
\hline & & Range & $22-53$ \\
\hline \multirow{2}{*}{ Years of education } & & Mean $\pm S D$ & $16.50 \pm 1.43$ \\
\hline & & Range & $12-19$ \\
\hline \multirow{2}{*}{ Sex } & Male & \multirow{2}{*}{ No. (\%) } & $31(62.0)$ \\
\hline & Female & & $19(38.0)$ \\
\hline \multirow{3}{*}{ Relationship status } & Single & & $36(72.0)$ \\
\hline & Married & No. (\%) & $13(26.0)$ \\
\hline & Divorced/ Widowed & & $1(2.0)$ \\
\hline
\end{tabular}

Table 2. Prevalence of UIT by category in participants

\begin{tabular}{cc}
\hline UIT Category & No. (\%) \\
\hline Contamination & $12(24)$ \\
\hline Harm & $14(28.0)$ \\
\hline Reubt & $40(80.0)$ \\
\hline Immoral & $10(20.0)$ \\
\hline Sexual & $10(20.0)$ \\
Victim & $5(10.0)$ \\
Others & $8(16.0)$ \\
\hline
\end{tabular}

CUNICAL PSYCH LOGY

Table 3. Means and standard deviations for ratings of the frequency, distress/interference, importance of removing, difficulty removing the MD-UITs by category

\begin{tabular}{ccccc}
\hline \multirow{2}{*}{ Variables } & \multicolumn{3}{c}{ Mean \pm SD } \\
\cline { 2 - 5 } & Frequency & Distress/Interference & Importance of Removing & Difficulty Removing \\
\hline Contamination & $3.00 \pm 1.13$ & $2.08 \pm 1.38$ & $3.17 \pm 1.47$ & $2.33 \pm 1.30$ \\
Harm & $2.21 \pm 0.89$ & $1.57 \pm 1.56$ & $3.21 \pm 1.48$ & $1.71 \pm 1.33$ \\
\hline Doubt & $3.13 \pm 1.24$ & $1.50 \pm 1.18$ & $2.33 \pm 1.38$ & $1.60 \pm 1.10$ \\
\hline Religious/immoral & $2.00 \pm 0.87$ & $1.71 \pm 1.57$ & $2.71 \pm 1.53$ & $1.71 \pm 1.05$ \\
\hline Sexual & $1.80 \pm 0.84$ & $1.20 \pm 1.10$ & $2.40 \pm 0.89$ & $1.60 \pm 1.14$ \\
Victim & $2.38 \pm 1.06$ & $2.00 \pm 1.07$ & $3.38 \pm 1.30$ & $2.25 \pm 1.49$ \\
Other & $2.68 \pm 1.06$ & $1.32 \pm 1.30$ & $2.53 \pm 1.31$ & $1.79 \pm 1.36$ \\
\hline
\end{tabular}


Table 4. Means and standard deviations of appraisal items

\begin{tabular}{cc}
\hline Appraisal & Mean \pm SD \\
\hline Overestimation of threat & $1.74 \pm 1.59$ \\
\hline Importance of thought & $2.98 \pm 1.33$ \\
\hline Intolerance of anxiety & $1.60 \pm 1.21$ \\
Need to control & $2.04 \pm 1.35$ \\
Responsibility & $2.10 \pm 1.49$ \\
\hline Intolerance of uncertainty & $1.36 \pm 1.69$ \\
\hline Perfectionism & $1.76 \pm 1.44$ \\
\hline Ego-dystonicity & $1.52 \pm 1.53$
\end{tabular}

Table 5. Means and standard deviations of control strategy items

\begin{tabular}{cl}
\hline Control Strategy & Mean \pm SD \\
\hline Distraction & $2.58 \pm 1.64$ \\
\hline Thought replacement & $2.60 \pm 1.57$ \\
\hline Thought stopping & $2.38 \pm 1.78$ \\
\hline Self- reassurance & $2.40 \pm 1.53$ \\
\hline Reassurance seeking & $0.88 \pm 1.27$ \\
\hline Ritualizing & $0.64 \pm 1.26$ \\
\hline Neutralization & $1.84 \pm 1.33$ \\
\hline Rationalization & $1.76 \pm 1.35$ \\
\hline Avoidance & $2.14 \pm 1.55$ \\
\hline
\end{tabular}

had the highest mean level for the importance of removing (Mean=3.38, $\mathrm{SD}=1.30$ ) and mean levels of interference/ distress (Mean=2.08, $\mathrm{SD}=1.38$ ), and difficulty removing (Mean=2.33, $\mathrm{SD}=1.30$ ) were higher in the contamination category. Mean ratings on the appraisal and control items are reported in Table 4 and Table 5, respectively. Participants reported responsibility (Mean=2.02, $\mathrm{SD}=1.29$ ), intolerance of uncertainty (Mean=2.10, $\mathrm{SD}=1.49)$, and anxiety (Mean=2.10, $\mathrm{SD}=1.49$ ) as the reasons that they noticed the thought. Participants were more likely to use thought replacement $($ Mean=2.60, $\mathrm{SD}=1.57)$, distraction (Mean=2.58, $\mathrm{SD}=1.64$ ), thought stopping (Mean=2.38, $\mathrm{SD}=1.78$ ), self-reassurance (Mean=2.40, $\mathrm{SD}=1.53$ ), avoidance (Mean=2.14, $\mathrm{SD}=1.55$ ) as thought control strategies. We also explored the difference in the prevalence of UIT endorsements by category between males and females and only found a statistically significant difference in immoral category $(32.30 \%$ in males versus $0.0 \%$ in females, $\mathrm{P}=0.008$ ).

\section{Discussion}

Cognitive models of obsessions and OCD were created mainly in the developed, English-speaking countries, and have rarely been studied cross-nationally or cross-culturally (Nedeljkovic et al., 2012; Radomsky et al., 2014). For example, little is known about the eastern culture, which is different from western culture in most similar ar- 
eas. There is no research information on the main effects of all these variables on the normal population. Thus, the main objectives of the present study were to examine three domains of OCD literature on the content, appraisals, and control strategies in the Iranian cultural context. Consistent with previous works (Radomsky et al., 2014), we found that, overall, $100 \%$ of the participants reported at least one type of unwanted intrusive thought at some point during the previous three months. While in Radomsky, et al. (2014) study, small differences were found in the nature and content of UITs. They indicated $100 \%$ of the participants from Fredericton, Montreal, and Tehran reported at least one UIT within the previous 90 days; only $81.2 \%$ of those from Thessaloniki reported UITs during the same period. In comparison with our findings, other studies have shown $79.8 \%-84 \%$ of repetitive, unwanted intrusive thoughts in the normal population $(\mathrm{Mu}-$ ris, Merckelbach, \& Clavan, 1997; Salkovskis, 1985). Although such findings might reflect significant international or cross-cultural differences, they merit further research (Radomsky et al., 2014).

One of the main objectives of the present study was to examine whether any of the six domains in IITIS as the nature and prevalence of intrusive thoughts are observed across the culture of the normal Iranian population. The highest prevalence of UITs was in the doubt category, and a large proportion of others' UITs were detected. However, the lowest prevalence of UITs was observed in the sex and then victim categories. The present findings on doubt and other categories are similar to Radomsky et al. (2014), but contrary to our finding on victim category, the least frequent UITs reported in their study belonged to the repugnant UITs like sex, immorality, and religion. The present finding to some extent is similar to other studies which reported the doubt category as the most prevalent one (Akhtar et al., 1975; Khanna et al., 1988; Rachman, 1998). The present finding in this study is also consistent with Shams et al. (2011), which found eight content categories in OCD with one of the main factors considered being checking compulsions. It has been suggested that normal and abnormal obsessions or intrusions are similar in form and content, but patients report more frequent, intense, and unacceptable, ego-dystonic, uncontrollable, and anxiety-provoking intrusions (Rachman \& de Silva, 1978).

The present finding on the priority of "doubt" and "other" intrusions as compared with other contexts of OCD (i.e. contamination, harm/injury/aggression, religious/ immoral, sexual, and victimization intrusions) in normal Iranian population needs further investigation and comparison with the findings of other studies (Alco- lado \& Radomsky, 2011; Ferrão et al., 2012; Gentsch, Schütz-Bosbach, Endrass, \& Kathmann, 2012; Ghisi, Chiri, Marchetti, Sanavio, \& Sica, 2010; Radomsky \& Alcolado, 2010). With regard to these findings, some theoretical implications may arise like whether doubting intrusions are markedly prevalent in non-clinical individuals. The "other" intrusions like miscellaneous category can be considered as the most common types of UITs endorsed in a non-clinical sample (Radomsky et al., 2014). However, the available research evidence casts doubt as to the universal cultural relevance of theories, asserting the important role of specific beliefs and appraisals in OCD symptoms.

The occurrence of unwanted and unacceptable intrusive thoughts or images initiates a process of faulty metacognitive appraisal and control efforts that can spiral into the development of obsessions. Later publications elaborating on a Cognitive Behavioral Therapy (CBT) model of obsessions and compulsions also reiterated the occurrence of mental intrusions as a key determinant in the pathogenesis of obsessions (Clark, 2004). Today the universality of obsession-related intrusive thoughts, images, and impulses is a central tenet of CBT explanations for the development of obsessions.

Generally, if we consider underlying beliefs and appraisals as intervening factors between obsessions and compulsions, which often play a role in preserving OCD, this question may arise that which domain of obsessive beliefs has a significant relation with OCD symptoms in the normal population. Our study found a high rate of intolerance of uncertainty, anxiety, and responsibility in the entire sample. The present findings on the intolerance of uncertainty, anxiety, and responsibility categories are similar to Radomsky et al. (2014) study findings. Still, contrary to our findings on the overestimation of threat, the importance of thoughts, and the need to control categories, the least frequent UITs reported in the present and Radomsky et al. (2014) study were perfectionism, ego-dystonicity, and thought-action fusion. The present finding on the importance of intolerance of uncertainty is also consistent with other studies (Holaway, Heimberg, \& Coles, 2006; Myers, Fisher, \& Wells, 2008; Shams \& Milosevic, 2015; Steketee, Frost, \& Cohen, 1998).

It has also been suggested that higher levels of intolerance of uncertainty are related to greater levels of psychological disturbance. Uncertainty can be considered as the only construct (compared to responsibility, control, threat estimation, tolerance for anxiety, and coping) that successfully predicts OCD symptoms above and beyond mood and worry (Steketee, Frost, \& Cohen, 1998). Gen- 
erally, the link between intolerance of uncertainty and symptoms of other disorders (depression, social anxiety disorder, post-traumatic stress disorder, eating disorders) suggests that intolerance uncertainty as a cognitive domain is highly related not only to OCD but also to other disorders (Abasi, Fata, Sadeghi, Banihashemi, \& Mohammadee, 2013; Gentes \& Ruscio, 2011). However, these constructs may play an integral role in the mental scene of the normal population and the development and maintenance of OCD, as well as several other disorders.

Nevertheless, this concept cannot be considered a part of cultural beliefs and attitudes, but rather an international pathological concept which not only appears in OCD, certain disorders or population but may also be considered as a global and universal concept. Still, the questions on cognitive domains remain to be answered. Future research in other countries and Iran needs to sift through this relationship.

One of the main goals of the current study was to examine the thought control strategies used by the normal Iranian population. We have employed IITIS as a measure for control strategies of unwanted thoughts. The results indicated that the normal Iranian population reported elevated levels of distraction, thought replacement, thought stopping, self-reassurance, and avoidance. However, in Iranian culture, the mentioned strategies are associated with higher levels of health. Nevertheless, more research is still needed in the Iranian population to determine whether the tendency to use these strategies is linked to mental health. This result raises the next question: to what extent these types of functional thought control strategies are characteristic and specific to the normal Iranian population. Except for rationalization, frequent use of distraction, thought replacement, thought stopping, self-reassurance, avoidance strategies in normal Iranian individuals is consistent with previous findings in normal individuals (Radomsky et al., 2014).

Meanwhile, findings regarding the use of avoidance have been less consistent with previous findings (Radomsky et al., 2014). Given the heightened use of certain strategies in normal Iranian individuals as compared with Radomsky et al. (2014), it is also interesting to consider the possible meaning of these strategies. However, like the present finding, other studies reported scores higher on distraction normal population than OCDs (Fehm \& Hoyer, 2004). OCD is associated with lower use of distraction strategy (Abramowitz et al., 2003; Rassin \& Diepstraten, 2003), and correlated with fewer psychological symptoms (Rassin \& Diepstraten, 2003). In our opinion, all of the Iranian results and the studies mentioned above offer con- clusive evidence about the specificity of certain thought control strategies in the normal population from different countries. Nevertheless, the results of the present research vary, and it has remained unclear whether the tendency toward distraction, thought replacement, thought stopping, self-reassurance, and avoidance is linked or specific to the normal Iranian population. Generally, these variables in the Iranian population, which seem to be a prevalent strategy to control unpleasant thoughts, need to be reconsidered in the future. The present research results should be interpreted considering its limitations, such as small sample size. Furthermore, the research sample was normal population, and as it is mentioned before, the nature and frequency of intrusive thoughts and their control strategies may be different in clinical population, so future research should consider these restrictions.

In summary, the initial findings in the present study raised some questions about whether any of the six domains of the intrusive thoughts and thought control strategies are observed across all cultures of the normal population in the world. How is it that some individuals are more prone to experience certain intrusive thoughts in some cultures than others? What type of cognitive domain and thought control are employed by individuals in different cultures? What are the critical variables that distinguish the cognitive domain and thought control strategy in other cultures? What role cognitive domains and thought control strategies play in the persistence of UITs in the normal population of different cultures? All these questions may be addressed in future studies.

\section{Conclusion}

The general findings of this study confirmed the certain specific nature and prevalence of intrusive thoughts in Iranian culture. The high rate of intolerance of uncertainty, anxiety, and responsibility in the Iranian sample may suggest that these constructs play an integral role in the native Iranian culture. This finding may raise the question of whether these constructs are the center of attraction in Iranian cultural beliefs and attitudes, or rather an international psychological concept which is observed not only in Iranian culture but in many other cultures, too. The finding on the higher prevalence of UITs in doubt category and the lower prevalence of UITs in sexual and victim categories needs further research in Iranian as well as other cultures. Overall, this study has highlighted certain concepts of cognitive domain and control strategy in the normal population as a representative of a healthy population that requires further research. With the preliminary studies on intrusive thoughts, it may be suggested that the previous OCD measures like 
VOCI (the Vancouver obsessive-compulsive inventory), OCI-R (obsessive-compulsive inventory-revised), PIWSU-R (Padua inventory Washington State University revision) and many other questionnaires assess only one aspect of OCD (like OC symptoms), while the IITIS contribute to measuring three main areas (OC symptoms UITs, cognitive domains, and control strategies).

It is better to review and finalize the differences in varieties of OC subscales presented in different OCD questionnaires like VOCI, OCI-R, PI-WSU-R, and add those subscales from these questionnaires to IITIS to make it a final OCD measure. Thus, the IITIS with this theoretical base of assessment has the potential to be considered as a standard and universal measurement in OCD assessment.

\section{Ethical Considerations}

\section{Compliance with ethical guidelines}

All ethical principles were considered in this article. The participants were informed about the purpose of the research and its implementation stages; they were also assured about the confidentiality of their information; Moreover, They were allowed to leave the study whenever they wish, and if desired, the results of the research would be available to them.

\section{Funding}

This work was supported by Tehran University of Medical Sciences (Grant Number: 96-01-30-33523).

\section{Authors' contributions}

Assisted with the design and completion of the study, read and revised the whole report: All authors; Analyzed the data: Giti Shams and Imaneh Abasi. Drafted the manuscript: Giti Shams and Imaneh Abasi.

\section{Conflict of interest}

The authors declared no conflicts of interest.

\section{References}

Abasi, I., Fata, L., Sadeghi, M., Banihashemi, S., \& Mohammadee, A. (2013). A comparison of transdiagnostic components in generalized anxiety disorder, unipolar mood disorder and non-clinical population. World Academy of Science, Engineering and Technology, International Journal of Medical,
Health, Biomedical, Bioengineering and Pharmaceutical Engineering, 7(12), 803-11.

Abramowitz, J. S. (2006). Understanding and treating obsessivecompulsive disorder: A cognitive behavioral approach. United Kingdom: Routledge. [DOI:10.4324/9781410615718]

Abramowitz, J. S., Franklin, M. E., Schwartz, S. A., \& Furr, J. M. (2003). Symptom presentation and outcome of cognitive-behavioral therapy for obsessive-compulsive disorder. Journal Of Consulting And Clinical Psychology, 71(6), 1049-56. [DOI:10.1037/0022-006X.71.6.1049] [PMID]

Akhtar, S., Wig, N., Varma, V., Pershad, D., \& Verma, S. (1975). A phenomenological analysis of symptoms in obsessive compulsive neurosis. The British Journal of Psychiatry, 127(4), 342-8. [DOI:10.1192/bjp.127.4.342] [PMID]

Alcolado, G. M., \& Radomsky, A. S. (2011). Believe in yourself: Manipulating beliefs about memory causes checking. Behaviour Research and Therapy, 49(1), 42-9. [DOI:10.1016/j. brat.2010.10.001] [PMID]

Belloch, A., Morillo, C., \& Garcia-Soriano, G. (2009). Strategies to control unwanted intrusive thoughts: Which are relevant and specific in obsessive-compulsive disorder? Cognitive Therapy and Research, 33(1), 75-89. [DOI:10.1007/s10608-0079141-2

Brown, G. P., \& Clark, D. A. (2014). Assessment in Cognitive Therapy. United State: Guilford Publications. [DOI:10.26530/ OAPEN 575253]

Clark, D. A. (2004). Cognitive-behavioral therapy for OCD. United Kingdom: Guilford Press.

Clark, D. A., \& Purdon, C. L. (1995). The assessment of unwanted intrusive thoughts: A review and critique of the literature. Behaviour Research and Therapy, 33(8), 967-76. [DOI:10.1016/0005-7967(95)00030-2]

Del-Porto, J. A. (2001). Epidemiologia e aspectos transculturais do transtorno obsessivo-compulsivo. Revista Brasileira de Psiquiatria, 23, 3-5. [DOI:10.1590/S1516-44462001000600002]

Dugas, M. J., Gosselin, P., \& Ladouceur, R. (2001). Intolerance of uncertainty and worry: Investigating specificity in a nonclinical sample. Cognitive Therapy and Research, 25(5), 551-8. [DOI:10.1023/A:1005553414688]

Fehm, L., \& Hoyer, J. (2004). Measuring thought control strategies: The thought control questionnaire and a look beyond. Cognitive Therapy and Research, 28(1), 105-17. [DOI:10.1023/ B:COTR.0000016933.41653.dc]

Ferrão, Y. A., Shavitt, R. G., Prado, H., Fontenelle, L. F., Malavazzi, D. M., \& de Mathis, M. A. (2012). Sensory phenomena associated with repetitive behaviors in obsessive-compulsive disorder: An exploratory study of 1001 patients. Psychiatry Research, 197(3), 253-8. [DOI:10.1016/j. psychres.2011.09.017] [PMID]

Freeston, M. H., \& Ladouceur, R. (1997). What do patients do with their obsessive thoughts? Behaviour Research and Therapy, 35(4), 335-48.

Gentes, E. L., \& Ruscio, A. M. (2011). A meta-analysis of the relation of intolerance of uncertainty to symptoms of generalized anxiety disorder, major depressive disorder, and obsessive-compulsive disorder. Clinical Psychology Review, 31(6), 923-33. [DOI:10.1016/j.cpr.2011.05.001] [PMID] 
Gentsch, A., Schütz-Bosbach, S., Endrass, T. \& Kathmann, N. (2012). Dysfunctional forward model mechanisms and aberrant sense of agency in obsessive-compulsive disorder. Biological Psychiatry, 71(7), 652-9. [DOI:10.1016/j.biopsych.2011.12.022] [PMID]

Ghisi, M., Chiri, L. R., Marchetti, I., Sanavio, E., \& Sica, C. (2010). In search of specificity: "Not just right experiences" and obsessive-compulsive symptoms in non-clinical and clinical Italian individuals. Journal of Anxiety Disorders, 24(8), 879-86. [DOI:10.1016/j.janxdis.2010.06.011] [PMID]

Glass, C. R., \& Arnkoff, D. B. (1997). Questionnaire methods of cognitive self-statement assessment. Journal Of Consulting And Clinical Psychology, 65(6), 911. [DOI:10.1037/0022006X.65.6.911] [PMID]

Grisham, J. R., \& Williams, A. D. (2009). Cognitive control of obsessional thoughts. Behaviour Research and Therapy, 47(5), 395-402. [DOI:10.1016/j.brat.2009.01.014] [PMID]

Holaway, R. M., Heimberg, R. G., \& Coles, M. E. (2006). A comparison of intolerance of uncertainty in analogue obsessive-compulsive disorder and generalized anxiety disorder. Journal of Anxiety Disorders, 20(2), 158-74. [DOI:10.1016/j. janxdis.2005.01.002] [PMID]

Khanna, S., Rajendra, P., \& Channabasavanna, S. (1988). Life events and onset of obsessive compulsive disorder. International Journal of Social Psychiatry, 34(4), 305-9. [DOI:10.1177/ 002076408803400408] [PMID]

Lipton, M. G., Brewin, C. R., Linke, S., \& Halperin, J. (2010). Distinguishing features of intrusive images in obsessivecompulsive disorder. Journal of Anxiety Disorders, 24(8), 81622. [DOI:10.1016/j.janxdis.2010.06.003] [PMID]

Moulding, R., Aardema, F., \& O'Connor, K. P. (2014). Repug nant obsessions: A review of the phenomenology, theoretical models, and treatment of sexual and aggressive obsessional themes in OCD. Journal of Obsessive-Compulsive and Related Disorders, 3(2), 161-8. [DOI:10.1016/j.jocrd.2013.11.006]

Muris, P., Merckelbach, H., \& Clavan, M. (1997). Abnormal and normal compulsions. Behaviour Research and Therapy, 35(3), 249-52. [DOI:10.1016/S0005-7967(96)00114-3]

Myers, S. G., Fisher, P. L., \& Wells, A. (2008). Belief domains of the Obsessive Beliefs Questionnaire-44 (OBQ-44) and their specific relationship with obsessive-compulsive symptoms. Journal of Anxiety Disorders, 22(3), 475-84. [DOI:10.1016/j. janxdis.2007.03.012] [PMID]

Nedeljkovic, M., Moulding, R., Foroughi, E., Kyrios, M., \& Doron, G. (2012). Cultural issues in understanding and treating obsessive compulsive and spectrum disorders. Oxford Handbook Of Obsessive Compulsive And Spectrum Disorders, 1-66. [Online Publication]. [DOI:10.1093/oxford$\mathrm{hb} / 9780195376210.013 .0102]$

Purdon, C., \& Clark, D. A. (1993). Obsessive intrusive thoughts in non-clinical subjects. Part I. Content and relation with depressive, anxious and obsessional symptoms. Behaviour Research and Therapy, 31(8), 713-20. [DOI:10.1016/00057967(93)90001-B]

Rachman, S. (1998). A cognitive theory of obsessions: Elaborations. Behaviour Research and Therapy, 36(4), 385-401. [DOI:10.1016/0005-7967(70)90058-6]
Rachman, S., \& de Silva, P. (1978). Abnormal and normal obsessions. Behaviour Research and Therapy, 16(4), 233-48. [DOI:10.1016/0005-7967(78)90022-0]

Radomsky, A. S., \& Alcolado, G. M. (2010). Don't even think about checking: Mental checking causes memory distrust. Journal of Behavior Therapy and Experimental Psychiatry, 41(4), 345-51. [DOI:10.1016/j.jbtep.2010.03.005] [PMID]

Radomsky, A. S., Alcolado, G. M., Abramowitz, J. S., Alonso, P., Belloch, A., \& Bouvard, M., et al. (2014). Part 1-You can run but you can't hide: Intrusive thoughts on six continents. Journal of Obsessive-Compulsive and Related Disorders, 3(3) 269-79. [DOI:10.1016/j.jocrd.2013.09.002]

Rasmussen, S. A., \& Tsuang, M. T. (1986). Clinical characteristics and family history in DSM-III obsessive-compulsive disorder. The American Journal of Psychiatry, 143(3), 317-22.

Rassin, E., \& Diepstraten, P. (2003). How to suppress obsessive thoughts. Behaviour Research and Therapy, 41(1), 97-103. [DOI:10.1016/S0005-7967(02)00043-8]

Rowa, K., \& Purdon, C. (2003). Why are certain intrusive thoughts more upsetting than others? Behavioural and Cognitive Psychotherapy, 31(01), 1-11. [DOI:10.1017/ S1352465803001024

Ruscio, A., Stein, D., Chiu, W., \& Kessler, R. (2010). The epidemiology of obsessive-compulsive disorder in the National Comorbidity Survey Replication. Molecular Psychiatry, 15(1) 53-63. [DOI:10.1038/mp.2008.94] [PMID] [PMCID]

Salkovskis, P. M. (1985). Obsessional-compulsive problems: A cognitive-behavioural analysis. Behaviour Research and Therapy, 23(5), 571-83. [DOI:10.1016/0005-7967(85)90105-6]

Salkovskis, P. M., Forrester, E., \& Richards, C. (1997). Cognitive-behavioural approach to understanding obsessional thinking. The British Journal of Psychiatry. Supplement, 35, 5363. [DOI:10.1192/S0007125000297900]

Shams, G., Kaviani, H., Esmaili, Y., Ebrahimkhani, N., \& Manesh, A. A. (2011). Psychometric Properties of the Persian Version of the Padua Inventory: Washington State University Revision (PI-WSUR). Iranian journal of psychiatry, 6(1), 12-8.

Shams, G., \& Milosevic, I. (2015). A comparative study of obsessive beliefs in obsessive-compulsive disorder, anxiety disorder patients and a normal group. Acta Medica Iranica, 53(5), 301-10.

Shooka, A., Al-Haddad, M., \& Raees, A. (1998). OCD in Bahrain: a phenomenological profile. International Journal of Social Psychiatry, 44(2), 147-54. [DOI:10.1177/00207640980440 0207] [PMID]

Sica, C., Coradeschi, D., Sanavio, E., Dorz, S., Manchisi, D., \& Novara, C. (2004). A study of the psychometric properties of the Obsessive Beliefs Inventory and Interpretations of Intrusions Inventory on clinical Italian individuals. Journal of Anxiety Disorders, 18(3), 291-307. [DOI:10.1016/S08876185(03)00013-6]

Steketee, G., Frost, R. O., \& Cohen, I. (1998). Beliefs in obsessive-compulsive disorder. Journal of Anxiety Disorders, 12(6), 525-37. [DOI:10.1016/S0887-6185(98)00030-9]

Tolin, D. F., Abramowitz, J. S., Brigidi, B. D., \& Foa, E. B. (2003) Intolerance of uncertainty in obsessive-compulsive disor- 
der. Journal of Anxiety Disorders, 17(2), 233-42. [DOI:10.1016/ S0887-6185(02)00182-2]

Williams, M. T., Turkheimer, E., Schmidt, K. M., \& Oltmanns, T. F. (2005). Ethnic identification biases responses to the Padua Inventory for obsessive-compulsive disorder. Assessment, 12(2), 174-85. [DOI:10.1177/1073191105275620] [PMID] 
This Page Intentionally Left Blank 